\title{
A Systematic Review of the Effectiveness of Rehabilitation Therapy to Treat People with Chronic Knee Pain Following A Total Knee Replacement.
}

Nathan Adam Johns ( $\square$ nathan.johns1@monash.edu )

Peninsula Health https://orcid.org/0000-0003-1685-1072

Justine Naylor

South Western Sydney Local Health District

Brinda Thirugnanam

Epworth HealthCare

Dean Mckenzie

Epworth HealthCare

Bernadette Brady

Liverpool Hospital

John Olver

Epworth HealthCare

Research article

Keywords: Chronic knee, surgery, rehabilitation therapy

Posted Date: October 9th, 2020

DOl: https://doi.org/10.21203/rs.3.rs-70032/v1

License: (9) (i) This work is licensed under a Creative Commons Attribution 4.0 International License.

Read Full License 


\section{Abstract}

\section{Background:}

Chronic knee pain after a total knee replacement has been estimated to affect 10 to $30 \%$ of patients and is related to dissatisfaction with surgery, reduced function and reduced quality of life. Rehabilitation is often prescribed in the subacute period post-operatively, but it may offer benefit to the increasing numbers of patients with chronic pain after their knee replacement. The aim of this systematic review is to evaluate the effectiveness of rehabilitation to improve pain, function and quality of life in people with chronic knee pain persisting for more than 3 months following a total knee replacement.

\section{Methods:}

The systematic review was conducted following PRISMA guidelines with a search of the online databases Ovid Medline, Embase via Ovid, CINAHL Plus, PsycINFO, Ovid Emcare and Proquest from their earliest date to July 12, 2020. The search criteria included English language randomised controlled trials of rehabilitation strategies in any setting to treat people with chronic knee pain, defined as knee pain persisting for more than 3 months, following a total knee replacement. Rehabilitation programs included exercise therapy, patient education, cognitive and mind-body strategies and self-management and excluded medication trials, procedural techniques and complementary therapies.

\section{Results:}

There were 254 abstracts screened for eligibility with 13 remaining for full-text screening. Following fulltext screening, there were no studies that met the eligibility criteria for evaluating rehabilitation therapy to treat chronic knee pain persisting for more than 3 months following a total knee replacement.

\section{Conclusion:}

Despite the high volume of knee replacement surgery and the high incidence of moderate to severe chronic pain ensuing, there is currently no evidence available that rehabilitation commencing three months after surgery can effectively treat chronic pain and disability following a total knee replacement.

\section{Background:}

There were over 56,000 primary total knee replacement procedures performed in Australia in $2018^{1}$ and almost 100,000 are performed annually in the United Kingdom ${ }^{2}$, principally for management of knee osteoarthritis.

Despite pain and loss of function being cited as the main reasons for patients to undergo a total knee replacement ${ }^{3}$, unfavourable pain outcomes (same, worse or minimally improved pain scores) more than 3 months after a total knee replacement can affect $8-26 \%$ of patients ${ }^{4}$. Other studies have found higher incidences of chronic pain at rates of $30 \%{ }^{5}$ and $55 \%{ }^{6}$. 
Chronic pain is defined as pain persisting or recurring for 3 months or longer, a time period thought sufficient for adequate tissue healing 7,8 . Its presence after a total knee replacement is associated with reduced function, poor sleep and psychological health ${ }^{9,10}$, reduced quality of life ${ }^{11}$ and ongoing use of medications such as NSAIDS or opioids ${ }^{12}$.

There is, however, little evidence to guide treatment for chronic knee pain following a total knee replacement with an earlier systematic review finding only a single randomized controlled trial using botulinum toxin with a small benefit on pain intensity observed ${ }^{13}$. Revision knee surgery for pain also does not appear to be the answer with one study finding that $47 \%$ of patients receiving this treatment reported severe pain 3 years after revision ${ }^{14}$.

Almost $90 \%$ of people in Australia initially receive a rehabilitation program following a total knee replacement (either home or hospital based), with only $5 \%$ still receiving ongoing hospital-based treatment at 3 months post-surgery ${ }^{15}$. This systematic review evaluates whether rehabilitation programs started 3 months after a total knee replacement can reduce pain, improve function and improve quality of life in people with persistent knee pain.

\section{Methods:}

The reporting of this systematic review is based on the PRISMA guidelines ${ }^{16}$. The protocol for this systematic review was registered prospectively with PROSPERO (ID number: 122684).

Eligibility criteria included randomised controlled trials in people, older than 18 years of age with persistent knee pain more than 3 months after a primary total knee replacement. Studies were limited to the English language.

Rehabilitation programs could include one or all of the following in a single or comprehensive program including training, exercises, compensatory strategies, education, support, counselling, mind-body and cognitive strategies, self-management, modifications to the environment and provision of resources and assistive technology. Whilst there is no set definition of rehabilitation, this is in keeping with a modern understanding ${ }^{17,18}$. To be eligible, the program needed to be primarily designed to improve knee pain in people with chronic knee pain after a total knee replacement. The therapy could be delivered via an information booklet, in person, via an electronic device (phone, computer or tablet), be group-based or individualised, in a hospital or rehabilitation facility as an inpatient or outpatient, in a private clinic or home-based.

Exclusion criteria included surgery, procedural techniques and injections. The use of medication, TENS, acupuncture, "natural" or complementary therapies and cryotherapy were only permitted if used within a rehabilitation therapy program but not if used individually.

The primary outcome was chronic knee pain defined as any description of pain persisting in the knee region for greater than 3 months following a total knee replacement and reported using a standardised 
measure of pain such as a patient-reported outcome measure or a visual analogue scale. This includes people with chronic post-surgical pain as defined by the ICD-11 which describes pain as "developing or increasing in intensity after a surgical procedure" ${ }^{19}$.

Secondary outcomes were function and quality of life assessed using a standardised patient-reported outcome measure or visual analogue scale.

A search of the following electronic databases was performed: Ovid Medline (1946-12 July, 2020), Embase via Ovid (1974-12 July, 2020), CINAHL Plus (to 12 July, 2020), PsycINFO (1806-12 July, 2020), Ovid Emcare (1995-12 July, 2020) and Proquest. A comprehensive strategy was used (see appendix A, B and $C$ below). A search of referenced articles of identified literature meeting the eligibility criteria was also conducted. Contact was made with one author to clarify the use of a pain scale ${ }^{20}$.

The abstracts were downloaded to Endnote and titles and abstracts were screened by NAJ assessing for eligibility criteria. The full text of potentially eligible articles was then assessed by 2 independent reviewers (NAJ, BT) using a standardised approach with the software program Covidence (Covidence systematic review software, Veritas Health Innovation, Melbourne, Australia. Available at www.covidence.org). Any disagreement concerning eligibility was discussed between NAJ and BT with a plan for a third reviewer (KF) to make the final decision if necessary.

Risk of bias in eligible papers was to be assessed using the Cochrane Collaboration guidelines ${ }^{21}$ and the software Covidence. The Covidence tool assesses 7 domains which include sequence generation, allocation concealment, blinding of participants and personnel for All outcomes, blinding of outcome assessors for All outcomes, incomplete outcome data for all outcomes, selective outcome reporting and other sources of bias. This uses descriptors such as low risk, high risk or unclear risk.

At the protocol stage, the plan for data extraction was to use Covidence with data items to include study source, study methods, participant characteristics, interventions used, outcome assessments and timing of outcomes, results and funding sources.

At the protocol stage, a meta-analysis was planned if 2 or more studies were found with outcome measures assessing pain. The data was to be exported from Covidence to Review Manager 5 ((RevMan) [Computer program], Version 5.3. Copenhagen: The Nordic Cochrane Centre, The Cochrane Collaboration, 2014) by NJ. Aggregate participant data was to be used to calculate the effect size of the rehabilitation interventions to reduce pain, improve function and quality of life and would include an assessment of heterogeneity. As the number of eligible studies was likely to be small, a narrative descriptive synthesis was thought to be required.

\section{Results:}

The primary electronic database search of CINAHL plus, Embase, Emcare, Medline and PsycINFO resulted in 372 publications being identified, 136 of which were duplicates. Of the 254 remaining abstracts, a total 
of 8 publications were found to be potentially eligible and were therefore retrieved for full-text screening. The Proquest search of conference proceedings returned 169 abstract titles of which 5 additional publications were found to be potentially eligible and identified for full text screening. Together this produced 13 potentially eligible publications.

Of the 13 publications, 7 were ineligible as they did not clearly commence more than three months after a total knee replacement ${ }^{22-29}$. Two publications were not randomised controlled trials ${ }^{30,31}$. One randomised controlled trial included the WOMAC subscale as a pain measure in the author's manuscript ${ }^{20}$ but this was not included in the final edition ${ }^{32}$.

Two publications reported findings from different time points of the same small randomised trial, at 3 and 12 months ${ }^{33,34}$. Whilst this trial was randomised and conducted more than three months post total knee replacement, it was not designed to treat pain after a knee replacement but rather to improve function and was thus excluded. The search therefore resulted in no eligible papers being included in the review (Fig. 1) ${ }^{16}$.

\section{Discussion:}

This systematic review was designed to search and evaluate rehabilitation therapies such as exercise, education, self-help, cognitive therapies and mindfulness to treat people with chronic knee pain present for more than 3 months following a total knee replacement. This review found no randomised controlled trials of rehabilitation specifically addressing this problem.

Only a single randomised controlled trial of rehabilitation to treat people 3 months after a knee replacement was found with evaluations at 3 months ${ }^{33}$ and 12 months ${ }^{33,34}$ after the program commenced. It was excluded from being eligible in this systematic review as it was not designed to treat pain, but rather to evaluate aquatic resistance exercise and its effects on mobility and strength. The authors included a pain measure and as it is the only rehabilitation trial starting more than 3 months after knee replacement, a brief summary is included below.

The trial enrolled 50 subjects aged 55 to 75 years (mean 66 years), 3 months or more (mean 9.6 months) after a primary, unilateral knee replacement in a twice weekly, 12-week, aquatic therapy program. The pain scores at baseline were mild only and not significantly different (mean 16.8 in training group, mean 17.0 in control group, $p=0.96$ ), Table 1 . Immediately post trial, the pain subscale scores were not significantly reduced or significantly different between groups (training group mean 13.0, control group mean 15.5, $p=$ $0.42)$, Table 2. This is not entirely surprising as the program was not designed to reduce pain. At 12 months, the difference in mean pain scores between the groups was again not significant (training group mean 13.3, control group mean 9.5, $p=0.14$ ), Table 3. WOMAC Function subscale scores were also not significantly different between groups at each time point. 
The effect of rehabilitation on pain after knee replacement has been evaluated by other studies at earlier time points. Pre-surgical rehabilitation was found to be ineffective in reducing pain after a total knee replacement ${ }^{35}$. A systematic review of physiotherapy, compared with no therapy, starting immediately after a total knee replacement, found evidence for only a small mean between-group difference in pain ${ }^{36}$. Another systematic review evaluated the effect of post-discharge rehabilitation and physiotherapy interventions in the first 3 months on the development of chronic pain after a total knee replacement ${ }^{37}$. When compared with usual care, they found that no particular treatment was more beneficial than usual care for reducing pain at 12 months post total knee replacement

A strength and novelty of this systematic review is the use of 3 months as the starting point following total knee replacement for assessing studies, as this is the time frame post-surgery or injury where ongoing pain is considered to be persistent or chronic ${ }^{38}$. In observational studies, there appears to be little improvement in mean pain scores after 3 months ${ }^{39}$ and it may be that there is no current therapy, medication or technique being used that is more effective in reducing pain beyond this time. As $70 \%$ or more of patients recover well with mild or no pain after a total knee replacement with current practices, studies conducted within the first 3 months may be under powered to detect a significant difference in pain due to a rehabilitation therapy intervention. Trials that enrol patients who have been identified as having persistent pain at 3 months, may lead to more significant results. The TRIO trial identified at risk patients at 6 weeks post knee replacement and prescribed an intervention of a "progressive functional rehabilitation protocol" but found non-significant differences in pain scores and Oxford Knee Scores at 12 months compared to the standard care group ${ }^{24}$.

One of the limitations of this systematic review, is the definition of rehabilitation and self-help used for the literature search. This definition was used for evaluating the literature, but rehabilitation is unlikely to be provided in isolation in clinical practice. There are likely to be simultaneous consultations with physicians (rehabilitation, pain, general practice) and orthopaedic surgeons who may simultaneously be providing education, recommending exercises, prescribing medication or performing investigations that may impact on the experience of pain.

Without any evidence available to guide treatment for people with persistent pain after total knee replacement, a multidisciplinary biopsychosocial pain rehabilitation program of education, judicious medication use, exercise and cognitive strategies based on a theoretical neuroscience framework could be used ${ }^{40}$.

\section{Conclusion}

Despite the high rates of chronic knee pain persisting for more than 3 months after a total knee replacement, this systematic review has not found any studies that have evaluated rehabilitation as a treatment for pain for this condition. This does not mean that rehabilitation is ineffective but rather that there is currently no direct evidence base to support its use as a treatment for pain in the chronic phase after knee replacement. This study has identified an area likely to benefit from future research, especially 
given the growing numbers of people with chronic knee pain and its negative impact on function and quality of life.

\section{Abbreviations}

WOMAC - The Western Ontario and McMaster Universities Osteoarthritis Index (WOMAC)

ICD- 11 - International Classification of Diseases Revision 11

TENS - Transcutaneous Electric Nerve Stimulation

NSAIDS - Non Steroidal Anti-Inflammatory Drugs

\section{Declarations}

Ethics approval and consent to participate

Not applicable

Consent for publication

Not applicable

\section{Availability of data and material}

All data generated or analysed during this study are included in this published article

Competing interests

The authors declare that they have no competing interests

\section{Funding}

No funding was received

\section{Author's contributions}

Nathan Johns (NAJ) and Brinda Thirugnanam (BT) participated in screening for eligibility

Nathan Johns wrote the article with the PhD supervision team consisting of John Olver, Justine Naylor, Dean Mckenzie and Bernadette Brady who provided input into the study protocol, design and provided critical analysis at all stages of production.

\section{Acknowledgements}


Nathan Johns has undertaken this start as part of a PhD at Monash University in collaboration with the Epworth Monash Rehabilitation Study Unit.

\section{References}

1. Registry AOANJ. Hip, Knee \& Shoulder Arthroplasty:2019 Annual Report. 2019. Adelaide: Australian Orthopaedic Association.

2. Board TNE. 16th Annual Report 2019. 2019. www.njrcentre.org.uk.

3. Gademan MG, Hofstede SN, Vliet Vlieland TP, et al. Indication criteria for total hip or knee arthroplasty in osteoarthritis: a state-of-the-science overview. BMC Musculoskelet Disord 2016; 17: 463. 2016/11/11. DOI: 10.1186/s12891-016-1325-z.

4. Beswick $A D$, Wylde V, Gooberman-Hill R, et al. What proportion of patients report long-term pain after total hip or knee replacement for osteoarthritis? A systematic review of prospective studies in unselected patients. BMJ Open 2012; 2: e000435. 2012/02/24. DOI: 10.1136/bmjopen-2011-000435.

5. Thomazeau Jp. Predictive Factors of Chronic Post-Surgical Pain at 6 Months Following Knee Replacement: Influence of Postoperative Pain Trajectory and Genetics. Pain Physician 2016; 19: E729-E741.

6. Lavand'homme PM, Grosu I, France MN, et al. Pain trajectories identify patients at risk of persistent pain after knee arthroplasty: an observational study. Clin Orthop Relat Res 2014; 472: 1409-1415. 2013/11/22. DOI: 10.1007/s11999-013-3389-5.

7. Treede RD, Rief W, Barke A, et al. A classification of chronic pain for ICD-11. Pain 2015; 156: 10031007. 2015/04/07. DOI: 10.1097/j.pain.0000000000000160.

8. Schug SA, Lavand'homme P, Barke A, et al. The IASP classification of chronic pain for ICD-11: chronic postsurgical or posttraumatic pain. Pain 2019; 160: 45-52. 2018/12/27. DOI: 10.1097/j.pain.0000000000001413.

9. Wylde V, Beswick A, Bruce J, et al. Chronic pain after total knee arthroplasty. EFORT Open Rev 2018; 3: 461-470. 2018/09/22. DOI: 10.1302/2058-5241.3.180004.

10. Phillips JR, Hopwood B, Stroud R, et al. The characterisation of unexplained pain after knee replacement. Br J Pain 2017; 11: 203-209. 2017/11/11. DOI: 10.1177/2049463717719774.

11. da Silva RR, Santos AA, de Sampaio Carvalho Junior J, et al. Quality of life after total knee arthroplasty: systematic review. Rev Bras Ortop 2014; 49: 520-527. 2015/08/01. DOI: 10.1016/j.rboe.2014.09.007.

12. Singh JA and Lewallen DG. Predictors of use of pain medications for persistent knee pain after primary Total Knee Arthroplasty: a cohort study using an institutional joint registry. Arthritis Res Ther 2012; 14: R248. 2012/11/20. DOI: 10.1186/ar4091.

13. Beswick AD, Wylde V and Gooberman-Hill R. Interventions for the prediction and management of chronic postsurgical pain after total knee replacement: systematic review of randomised controlled trials. BMJ Open 2015; 5: e007387. 2015/05/15. DOI: 10.1136/bmjopen-2014-007387. 
14. Petersen KK, Simonsen O, Laursen MB, et al. Chronic postoperative pain after primary and revision total knee arthroplasty. The Clinical journal of pain 2015; 31: 1-6. 2014/12/09. DOI:

10.1097/AJP.0000000000000146.

15. Naylor JM, Hart A, Harris IA, et al. Variation in rehabilitation setting after uncomplicated total knee or hip arthroplasty: a call for evidence-based guidelines. BMC Musculoskelet Disord 2019; 20: 214. 2019/05/17. DOI: 10.1186/s12891-019-2570-8.

16. Moher D, A L, J T, et al. Preferred Reporting Items for Systematic Reviews and Meta-Analyses-The PRISMA Statement. PLoS Medicine 2009; 6. DOI: 10.1371/.

17. Wade DT. What is rehabilitation? An empirical investigation leading to an evidence-based description. Clinical rehabilitation 2020; 34: 571-583. 2020/02/11. DOI:

10.1177/0269215520905112.

18. Wade D. Rehabilitation--a new approach. Overview and Part One: the problems. Clinical rehabilitation 2015; 29: 1041-1050. 2015/10/16. DOI: 10.1177/0269215515601174.

19. Organisation WH. ICD-11, International Classification of Diseases for Mortality and Morbidity Statistics, Eleventh Edition, (2018, accessed 3/8/19 2019).

20. Piva SR, Almeida GJ, Gil AB, et al. A comprehensive behavioral and exercise intervention improves physical function and activity participation after total knee replacement - a pilot randomized study. Arthritis Care Res (Hoboken) 2017; 69: 1855-1862. 2017/02/22. DOI: 10.1002/acr.23227.

21. Higgins JPT GSe. Cochrane Handbook for Systematic Reviews of Interventions In: (editors) HJaGS, (ed.). www.handbook.cochrane.org: The Cochrane Collaboration, 2011.

22. Christiansen MB, Thoma LM, Master $\mathrm{H}$, et al. Preliminary findings of a novel physical therapist administered physical activity intervention after total knee replacement. Osteoarthritis and Cartilage 2018; Conference: 2018 Osteoarthritis Research Society International, OARSI World Congress. United Kingdom. 2026 (Supplement 2011) (pp S2334). Conference Abstract.

23. Fung $\mathrm{V}$, Ho A, Shaffer J, et al. Use of Nintendo Wii Fit in the rehabilitation of outpatients following total knee replacement: a preliminary randomised controlled trial. Physiotherapy 2012; 98: 183-188. Randomized Controlled Trial Research Support, Non-U.S. Gov't 2012/08/18. DOI: 10.1016/j.physio.2012.04.001.

24. Hamilton D, Beard D, Barker K, et al. Targeting physiotherapy to patients at risk of poor outcomes following total knee arthroplasty: the TRIO randomised controlled trial. Physiotherapy 2019; 105 : e160-e161. Conference Abstract. DOI: 10.1016/j.physio.2018.11.162.

25. Kauppila A, Kyllonen E, Ohtonen P, et al. Multidisciplinary rehabilitation after primary total kneearthroplasty: a randomized controlled study of its effects on functional capacity and quality of life. Clinical rehabilitation 2010; 24: 398-411.

26. Li L, Cheng S, Wang G, et al. Tai chi chuan exercises improve functional outcomes and quality of life in patients with primary total knee arthroplasty due to knee osteoarthritis. Complement Ther Clin Pract 2019; 35: 121-125. Article 2019/04/21. DOI: 10.1016/j.ctcp.2019.02.003. 
27. Moffet H, Collet JP, Shapiro SH, et al. Effectiveness of intensive rehabilitation on functional ability and quality of life after first total knee arthroplasty: A single-blind randomized controlled trial. Arch Phys Med Rehabil 2004; 85: 546-556. 2004/04/15. DOI: 10.1016/j.apmr.2003.08.080.

28. Piva SR, Gil AB, Almeida GJ, et al. A balance exercise program appears to improve function for patients with total knee arthroplasty: a randomized clinical trial. Phys Ther 2010; 90: 880-894. 2010/04/10. DOI: 10.2522/ptj.20090150.

29. Sadoghi P, Hasenhutl S, Gruber G, et al. Impact of a new cryotherapy device on early rehabilitation after primary total knee arthroplasty (TKA): a prospective randomised controlled trial. Int Orthop 2018; 42: 1265-1273. Article 2018/01/23. DOI: 10.1007/s00264-018-3766-5.

30. Chughtai M, Newman JM, Sultan AA, et al. The Role of Virtual Rehabilitation in Total Knee and Hip Arthroplasty. Surg Technol Int 2018; 32: 299-305. Article 2018/03/23.

31. Clatworthy M. Total Knee Replacement Plus Nonsurgical Treatment Was Better Than Nonsurgical Treatment Alone for Knee Osteoarthritis. J Bone Joint Surg Am 2016; 98: 873. Note 2016/05/20. DOI: 10.2106/JBJS.16.00208.

32. Piva SR, Almeida GJ, Gil AB, et al. Effect of Comprehensive Behavioral and Exercise Intervention on Physical Function and Activity Participation After Total Knee Replacement: A Pilot Randomized Study. Arthritis Care Res (Hoboken) 2017; 69: 1855-1862. 2017/02/22. DOI: 10.1002/acr.23227.

33. Valtonen A, Poyhonen T, Sipila $S$, et al. Effects of aquatic resistance training on mobility limitation and lower-limb impairments after knee replacement. Arch Phys Med Rehabil 2010; 91: 833-839. 2010/06/01. DOI: 10.1016/j.apmr.2010.03.002.

34. Valtonen A, Poyhonen T, Sipila S, et al. Maintenance of aquatic training-induced benefits on mobility and lower-extremity muscles among persons with unilateral knee replacement. Arch Phys Med Rehabil 2011; 92: 1944-1950. 2011/12/03. DOI: 10.1016/j.apmr.2011.07.191.

35. Chesham RA and Shanmugam S. Does preoperative physiotherapy improve postoperative, patientbased outcomes in older adults who have undergone total knee arthroplasty? A systematic review. Physiother Theory Pract 2017; 33: 9-30. Meta-Analysis Review Systematic Review 2016/10/14. DOI: 10.1080/09593985.2016.1230660.

36. Artz N, Elvers KT, Lowe CM, et al. Effectiveness of physiotherapy exercise following total knee replacement: systematic review and meta-analysis. BMC Musculoskelet Disord 2015; 16: 15. 2015/04/19. DOI: 10.1186/s12891-015-0469-6.

37. Wylde V, Dennis J, Gooberman-Hill R, et al. Effectiveness of postdischarge interventions for reducing the severity of chronic pain after total knee replacement: systematic review of randomised controlled trials. BMJ Open 2018; 8: e020368. 2018/03/02. DOI: 10.1136/bmjopen-2017-020368.

38. Apkarian AV. Definitions of nociception, pain, and chronic pain with implications regarding science and society. Neurosci Lett 2019; 702: 1-2. 2018/12/07. DOI: 10.1016/j.neulet.2018.11.039.

39. Naylor JM, Harmer AR, Heard RC, et al. Patterns of recovery following knee and hip replacement in an Australian cohort. Australian health review : a publication of the Australian Hospital Association 
2009; 33: 124-135. 2009/02/11. DOI: 10.1071/ah090124.

40. Lotze M and Moseley GL. Theoretical Considerations for Chronic Pain Rehabilitation. Phys Ther 2015; 95: 1316-1320. 2015/04/18. DOI: 10.2522/ptj.20140581.

\section{Tables}

Table 1: WOMAC pain and function subscales at baseline

\begin{tabular}{|lccccccc|}
\hline \multicolumn{5}{|c|}{ Pain subscale } & \multicolumn{5}{c|}{ Function subscale } \\
\hline & Number & Mean & $\begin{array}{l}\text { Standard } \\
\text { deviation }\end{array}$ & $\begin{array}{l}95 \% \\
\text { confidence } \\
\text { intervals }\end{array}$ & Mean & $\begin{array}{l}\text { Standard } \\
\text { deviation }\end{array}$ & $\begin{array}{l}95 \% \\
\text { confidence } \\
\text { interval }\end{array}$ \\
\hline $\begin{array}{l}\text { Training } \\
\text { Group }\end{array}$ & 25 & 16.8 & 10.6 & $12.4-21.2$ & 22.6 & 11.7 & $17.8-27.4$ \\
\hline $\begin{array}{l}\text { Control } \\
\text { Group }\end{array}$ & 21 & 17.0 & 14.6 & $10.4-23.6$ & 17.0 & 11.5 & $16.5-23.6$ \\
\hline
\end{tabular}

- Pain subscale group difference $=-0.2$, standard error $=3.7,95 \%$ confidence interval $=-7.7-7.3, p=$ 0.96

- Function subscale group difference $=5.6$, standard error $=3.4,95 \%$ confidence interval $=-1.3-12.5$, $p=0.11$

Table 2: WOMAC pain and function subscale results at 3 months

\begin{tabular}{|lccccccc|}
\hline \multicolumn{9}{|c|}{ Pain subscale } & \multicolumn{5}{c|}{ Function subscale } \\
\hline & Number & Mean & $\begin{array}{l}\text { Standard } \\
\text { deviation }\end{array}$ & $\begin{array}{l}95 \% \\
\text { confidence } \\
\text { intervals }\end{array}$ & Mean & $\begin{array}{l}\text { Standard } \\
\text { deviation }\end{array}$ & $\begin{array}{l}95 \% \\
\text { confidence } \\
\text { intervals }\end{array}$ \\
\hline $\begin{array}{l}\text { Training } \\
\text { Group }\end{array}$ & 25 & 13.0 & 8.7 & $9.4-16.6$ & 18.5 & 9.4 & $14.6-22.4$ \\
$\begin{array}{l}\text { Control } \\
\text { Group }\end{array}$ & 21 & 15.5 & 12.4 & $9.9-21.1$ & 17.3 & 17.2 & $14.0-22.0$ \\
\hline
\end{tabular}


- Pain subscale group difference $=-2.5$, standard error $=3.1,95 \%$ confidence interval $=-8.8-3.8, p=$ 0.42

- Function subscale group difference $=1.2$, standard error $4.0,95 \%$ confidence interval $=-6.9-9.3, p=$ 0.77

Table 3: WOMAC pain and function subscale results at 12 months

\begin{tabular}{|c|c|c|c|c|c|c|c|}
\hline & \multirow[b]{2}{*}{ Number } & \multicolumn{3}{|c|}{ Pain subscale } & \multicolumn{3}{|c|}{ Function subscale } \\
\hline & & Mean & $\begin{array}{l}\text { Standard } \\
\text { deviation }\end{array}$ & $\begin{array}{l}95 \% \\
\text { confidence } \\
\text { intervals }\end{array}$ & Mean & $\begin{array}{l}\text { Standard } \\
\text { deviation }\end{array}$ & $\begin{array}{l}95 \% \\
\text { confidence } \\
\text { intervals }\end{array}$ \\
\hline $\begin{array}{l}\text { Training } \\
\text { Group }\end{array}$ & 25 & 13.3 & 8.9 & $9.6-17.0$ & 18.1 & 11.0 & $13.6-22.6$ \\
\hline $\begin{array}{l}\text { Control } \\
\text { Group }\end{array}$ & 17 & 9.5 & 6.8 & $6.0-13.0$ & 14.8 & 11.0 & $9.1-20.5$ \\
\hline
\end{tabular}

- Pain subscale group difference $=3.8$, standard error $=2.6,95 \%$ confidence interval $=-1.4-9.0, p=$ 0.14

- Function subscale group difference $=3.3$, standard error $=3.5,95 \%$ confidence interval $=-3.7-10.2$, $\mathrm{p}=0.35$

Figures 


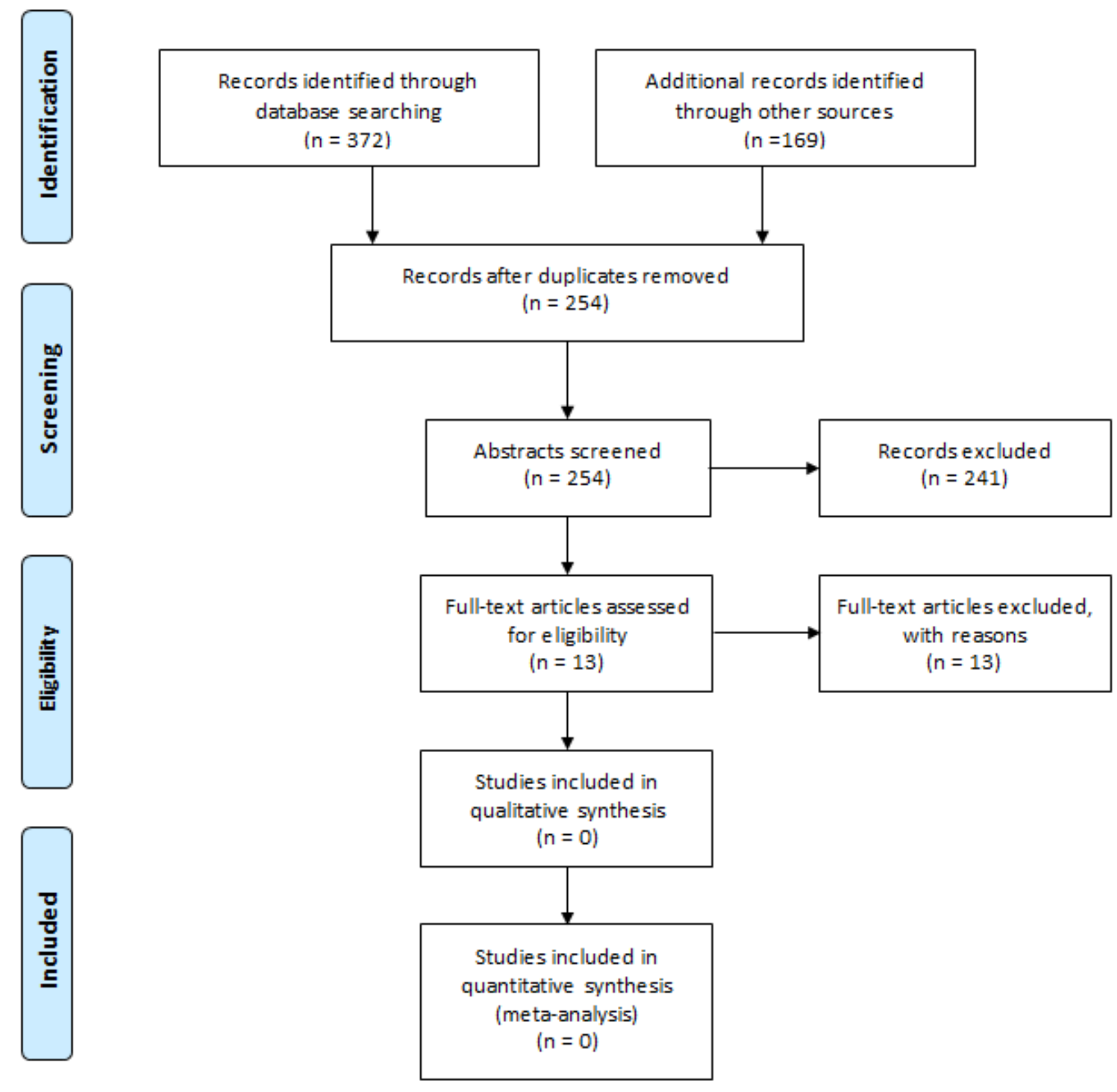

Figure 1

PRISMA 2009 Flow Diagram

\section{Supplementary Files}

This is a list of supplementary files associated with this preprint. Click to download.

- Appendix.docx 